\title{
Three-dimensional acoustic energy flow from rotating sound sources
}

\author{
Christof Ocker* (1) and Wolfram Pannert \\ Aalen University of Applied Sciences, 73430 Aalen, Germany
}

Received 26 July 2021, Accepted 22 October 2021

\begin{abstract}
A detailed study of the three-dimensional sound fields around a monopole point source in subsonic and supersonic rotation is investigated. We calculate the sound pressure, the acoustic velocity, as well as the instantaneous and time-averaged acoustic intensity fields with the advanced time approach. The advanced time approach is applied for sound sources at subsonic and supersonic rotation and yield comparable results as those obtained from the spherical harmonic series expansion method or the solution of the retarded time equation. Further, the direction of the time-averaged acoustic energy flow is derived from the acoustic intensity vectors. It is shown, that the direction of the energy flow path differs from the radial direction and depends on the rotational direction and rotational velocity. Those findings are useful, for example, to improve the acoustic absorption performance of sound absorbers and acoustic liners around turbomachines.
\end{abstract}

Keywords: Rotating sound sources, Supersonic rotational velocity, Acoustic intensity field, Advanced time approach

\section{Introduction}

Turbomachines, like axial fans or aircraft engines, are often used in direct vicinity of humans due to their applications in transportation, cooling and air-conditioning. However, they are among the system components with the highest sound generation. Hence, the sound field due to rotating sources in turbomachines is of great interest, especially, in terms of sound absorbers. The calculation of the sound field and the acoustic energy flow may help to develop and to improve the acoustic absorption performance of micro-perforated duct absorber of axial fans [1-3] and acoustic liners, which were integrated into the casing around an aircraft engine $[4,5]$. For those absorbers, the best absorption performance is obtained if the angle of the acoustic intensity vector corresponds to the normal direction of the absorbing surface. Therefore, the sound pressure field, the acoustic velocity field, and the instantaneous and time-averaged active acoustic intensity fields due to a rotating sound source need to be calculated. For the calculation of the pressure field due to a rotating monopole point source, mainly three methods were developed: the Spherical Harmonic Series Expansion Method (SHSEM) [6, 7], the advanced time approach [8] and the retarded time approach $[9,10]$. Poletti and Teal [11] evaluated the sound pressure due to a rotating monopole at a specific observer position for sound sources rotating at subsonic and supersonic velocities and compared the three methods to each other. For the subsonic case, all three

*Corresponding author: christof .ocker@hs-aalen.de methods produce a comparable waveform at the observer position. For the supersonic case, only the SHSEM and the retarded time approach were used to evaluate the sound pressure. The main findings of their work are: first, the advanced time approach could not be used for the supersonic case due to aliasing effects. Second, the calculation of the sound pressure for the supersonic case is computationally expensive with the SHSEM due to the high mode order, which is needed to reconstruct the waveform. Further, this method is band-limited due to the truncation of the series expansion. Third, the retarded time approach is applicable for Mach numbers greater than 1. However, using the retarded time formulation gets more challenging for the supersonic case, because multiple roots of a complex nonlinear equation have to be calculated to take all wave fronts into account, which arrive at the receiver position at the same time. The acoustic velocity and intensity field were not investigated in their work. Mao et al. [12] were the first we are aware of, who calculated the instantaneous and time-averaged acoustic intensity fields around rotating sound sources. In their work, the sound pressure due to a rotating monopole and dipole was calculated with the frequency-domain numerical method [13-15]. However, the frequency-domain numerical method is only efficient for tonal noise prediction. Considering broadband noise sources, the numerical integration has to be repeated for each frequency [16]. Hence, only one frequency component of the Doppler-shifted spectrum due to a rotating monochromatic point source was considered in their investigation. Further, the sound sources rotated only at subsonic velocity. 
The focus of the present paper is to calculate the threedimensional sound fields around a rotating source with moderate numerical effort. We visualize the sound pressure field, the acoustic velocity field and the instantaneous and time-averaged active acoustic intensity fields around sound sources rotating at subsonic and supersonic velocities. By considering high sampling rates of up to $f_{\mathrm{s}}=1 \times 10^{6} \mathrm{~Hz}$ and a low-pass Butterworth filter, the waveform of the pressure signal is reconstructed well for the subsonic and the supersonic case using the advanced time approach. Revisiting the advanced time approach, hence, provides an easy to implement and straightforward numerical sound prediction method of the acoustic energy flow of sound sources at subsonic and supersonic rotation. Section 2 reviews the advanced time approach and the calculation of the instantaneous and time-averaged acoustic intensity. In Section 3, the three-dimensional sound pressure, acoustic velocity and acoustic intensity fields due to a rotating sound source are calculated for the subsonic and supersonic case. Therefore, the test cases of Poletti and Teal [11] are investigated and extended. Section 4 draws some conclusions.

\section{Theory}

The evaluation of the sound pressure $p(\mathbf{x}, t)$ with the advanced time approach starts with the frequency-domain Green's function for a stationary monopole sound source

$$
p(\mathbf{x}, t)=\frac{e^{-j k r}}{4 \pi r} e^{j \omega_{0} t},
$$

$r=\left|\mathbf{x}-\mathbf{x}_{\mathrm{s}}\right|$ is the distance between the observer position $\mathbf{x}$ and the source position $\mathbf{x}_{\mathrm{s}}, k=\omega_{0} / c_{0}$ is the wave number, $\omega_{0}$ is the angular frequency of the source pulsation, $c_{0}$ is the speed of sound and $t=\left|\mathbf{x}-\mathbf{x}_{\mathrm{s}}\right| / c_{0}$ is the traveling time.

In general, the acoustic velocity vector is calculated from the linearized momentum equation

$$
\rho_{0} \frac{\partial \mathbf{u}(\mathbf{x}, t)}{\partial t}=-\nabla p(\mathbf{x}, t)
$$

In the spherical coordinate system, the gradient of the pressure field depends only on the radial distance $r$

$$
\frac{\partial}{\partial r} p(\mathbf{x}, t)=-\frac{e^{-j k r}(j k r+1)}{4 \pi r^{2}} e^{j \omega_{0} t}
$$

Considering the harmonic acoustic velocity in radial direction $u_{r}(\mathbf{x}) e^{j \omega_{0} t}$, Equation (2) can be rewritten as

$$
u_{r}(\mathbf{x}) \frac{\partial}{\partial t} e^{j \omega_{0} t}=\frac{1}{\rho_{0}} \frac{e^{-j k r}(j k r+1)}{4 \pi r^{2}} e^{j \omega_{0} t} .
$$

Calculating the time derivative of $e^{j \omega_{0} t}$ and inserting Equation (1) into Equation (4) leads to

$$
u_{r}(\mathbf{x}) e^{j \omega_{0} t}=\frac{1}{j \omega_{0} \rho_{0}} \frac{e^{-j k r}(j k r+1)}{4 \pi r^{2}} e^{j \omega_{0} t}=\frac{p(\mathbf{x}, t)}{Z_{0}}\left(1-\frac{j}{k r}\right),
$$

where $Z_{0}=\rho_{0} c_{0}$ is the specific acoustic impedance and $\rho_{0}$ the density of the fluid. For a monopole point source, the other spherical components of the acoustic velocity are zero. To consider the movement of the sound source, the advanced time approach can be applied on the pressure signal and the acoustic velocity signal separately.

Considering a rotating monopole sound source emitting a harmonic acoustic signal. For the advanced time approach, the pressure signal and the acoustic velocity signal at the observer position were obtained from the summation of all acoustic signals at those times they reach the observer position. The advanced time approach is similar to the retarded time approach, but from the point of view of the sound source. An acoustic signal is emitted from the source position $\mathbf{x}_{\mathrm{s}}$ at emission time $t_{\mathrm{e}}$ and travels at speed of sound $c_{0}$ until it reaches the observer position $\mathbf{x}$ at the distance $r$. Due to the movement of the sound source, the acoustic signal reaches the observer position at the advanced times

$$
t_{\mathrm{adv}}=t_{\mathrm{e}}+\frac{\left|\mathbf{x}-\mathbf{x}_{\mathrm{s}}\left(t_{\mathrm{e}}\right)\right|}{c_{0}} .
$$

The time spacing between samples of the advanced time signals for the pressure and the acoustic velocity are distributed non-uniformly and the data samples at the advanced times $t_{\mathrm{adv}}$ are in general not multiples of a fixed time delay $\Delta t$. Hence, the acoustic signals at the observer must be interpolated. Typically, a linear interpolation or a resampling is recommended for subsonic rotational velocities before further processing (e.g., a Fast Fourier Transformation, FFT). However, in the following analysis, a sinc interpolation [17] is used, which provides a superior reconstruction of the resulting irregular time signals. Further, the sinc interpolation is exact for a band-limited signal in the subsonic case and uniform sampling rates. Maymon and Oppenheim [18] applied different versions of the sinc interpolation and found a good signal reconstruction performance even on non-uniform distributed samples. In this work, a normalized time-windowed sinc function was used to calculate the sound pressure at the observer position $p_{\text {adv }}\left(\mathbf{x}, t_{\text {adv }}\right)$ from the sound pressure at the source position at emission time $p\left(\mathbf{x}_{\mathrm{s}}, t_{\mathrm{e}}\right)$

$$
p_{\mathrm{adv}}(k)=\sum_{l=n-L}^{n+L} p(l) \frac{\sin \left(\pi f_{\mathrm{s}}\left(k \Delta t-t_{\mathrm{adv}}(l \Delta t)\right)\right)}{\pi f_{\mathrm{s}}\left(k \Delta t-t_{\mathrm{adv}}(l \Delta t)\right)},
$$

where $k \Delta t$ are the uniform distributed time samples at the observer position and $l \Delta t$ at the source position. $f_{\mathrm{s}}=\frac{1}{\Delta t}$ is the sampling frequency and $L$ is set to 25 to limit the sinc interpolation to $2 L+1$ samples. Then, the sinc interpolation is applied to the acoustic velocity amplitude in the same way. The acoustic velocity vector $\mathbf{u}(\mathbf{x}, t)$ results from the superposition of the contributions from the different source positions in the range $k \in[n-L, n+L]$.

The acoustic intensity is then calculated with the sinc interpolated sound pressure and acoustic velocity vector. The instantaneous acoustic intensity vector IAI $\mathbf{I}(\mathbf{x}, t)$ is calculated from the real parts of the fluctuating sound pressure $p$ and the acoustic velocity vector $\mathbf{u}$ as

$$
\mathbf{I}(\mathbf{x}, t)=\operatorname{Re}(p(\mathbf{x}, t)) \operatorname{Re}(\mathbf{u}(\mathbf{x}, t)) .
$$


The time-averaged acoustic intensity AAI $\overline{\mathbf{I}}(\mathbf{x})$ is defined as the integral of the IAI over the time period of the acoustic signal $T$ as

$$
\overline{\mathbf{I}}(\mathbf{x})=\frac{1}{T} \int_{0}^{T} \mathbf{I}(\mathbf{x}, t) \mathrm{d} t,
$$

where $T$ should be a multiple of the source rotation period.

The frequency components of the time-averaged acoustic intensity $\tilde{\mathbf{I}}(\mathbf{x}, \omega)$ are calculated by applying the multiplicative theorem of the Fourier transform for a specific angular frequency $\omega$ as

$$
\tilde{\mathbf{I}}(\mathbf{x}, \omega)=\frac{p(\mathbf{x}, \omega) \mathbf{u}^{*}(\mathbf{x}, \omega)}{2},
$$

where the asterisk denotes the conjugate of the complex quantity. In general, the acoustic intensity $\tilde{\mathbf{I}}(\mathbf{x}, \omega)$ is complex valued. The real part of Equation (10) is the timeaveraged active acoustic intensity and represents the time-averaged acoustic energy flow for a specific frequency component. The imaginary part is the time-averaged reactive intensity [19]. Since the acoustic energy flow is contributed only from the active acoustic intensity, the reactive intensity is not considered in the following analysis. The integration over the angular frequency $\omega$ for the components of the active acoustic intensity $\tilde{\mathbf{I}}(\mathbf{x}, \omega)$ leads again to the time-averaged acoustic intensity $\overline{\mathbf{I}}(\mathbf{x})$.

\section{Sound fields around a rotating point source}

In this section, we calculate the sound fields around a rotating monopole point source in subsonic and supersonic rotation. Further, the angle of the acoustic energy flow relative to the radial direction is calculated and discussed for different rotational velocities and distances from the sound source.

\subsection{Subsonic rotation}

Poletti and Teal [11] investigated a monopole point source with a steady unit source strength, which rotates in counter-clockwise direction around the $z$-axis at a radius of $\rho_{\mathrm{s}}=1 \mathrm{~m}$ on the $x y$-plane. The observer is located at the position $(x, y, z)=(2 \mathrm{~m}, 0 \mathrm{~m}, 0 \mathrm{~m})$. For the subsonic case, the point source rotates with a rotation frequency of $f_{\mathrm{R}}=$ $27 \mathrm{~Hz}$ and emits a monochromatic signal at the frequency $f_{0}=500 \mathrm{~Hz}$. The rotational Mach number is $M_{0}=$ $2 \pi f_{\mathrm{R}} \rho_{\mathrm{s}} / c_{0} \approx 0.5$. The Doppler effect due to the rotation of the sound source yields a line spectrum with the frequency components $f_{0}+m f_{\mathrm{R}}$, where the mode number $m$ is an integer number. Positive values for $m$ give the modes which rotate in the same direction as the sound source and negative values are modes which rotate in the counter direction.

In Figure 1, the pressure time signal and the pressure spectrum at the observer position are calculated with the advanced time approach and compared to the results from Poletti and Teal [11]. For the evaluation of the time signal, the source data of 27 periods of rotation and a frequency sampling of $f_{\mathrm{s}}=2.7 \times 10^{5} \mathrm{~Hz}$ are used. Especially for high Mach numbers, a high frequency sampling is needed to reconstruct the waveform correctly. Due to the advanced time approach, the sampling rate of the advanced time signal is non-uniformly distributed. To obtain the pressure spectrum via a FFT, first, a uniform sampled time signal is generated using sinc interpolation. Then, a Kaiser window with the fifth-order was applied to the time signal before taking the FFT [20]. The resulting frequency resolution is $1 \mathrm{~Hz}$.

The pressure signal and the pressure spectrum agree well with those obtained by Poletti and Teal [11]. The biased errors between both methods are negligible.

As a second step, the acoustic pressure field is calculated by applying the advanced time approach for different observer positions. The observed plane is discretized with an equally-spaced Cartesian grid consisting of $21 \times 21$ points with $0.2 \mathrm{~m}$ spacing. The resulting area is $4 \mathrm{~m} \times 4 \mathrm{~m}$. First, we calculate the instantaneous acoustic velocity vectors following Equation (5), and further, the IAI $\mathbf{I}(\mathbf{x}, t)$ from the instantaneous acoustic pressure and the instantaneous acoustic velocity vector following Equation (8).

Figure 2 shows the contour of the instantaneous acoustic pressure relative to $p_{\text {ref }}=20 \mu \mathrm{Pa}$ with a dynamic range of $50 \mathrm{~dB}$. The sound pressure field displays the expected compression of the wave fronts in the forward and expansion in the reverse direction that is typical for a moving sound source. The arrows of uniform length indicate the vector direction of the instantaneous acoustic velocity vectors at $t=0$. The vector of the acoustic velocity points in the direction of the negative acoustic pressure gradient (see Eq. (2)).

Figure 3 shows the contour of the IAI level relative to $I_{\text {ref }}=10^{-12} \mathrm{Wm}^{-2}$. The arrows give the vector direction of the IAI at $t=0 \mathrm{~s}$. Most of the acoustic energy flows out of the sound source. However, in the region near the sound source, the energy flows into the point source (see Fig. 3b). In the vicinity of the sound source, the phase shift between the sound pressure and the acoustic velocity may be large (see Eq. (5)). Hence, only a small part of energy propagates into the far-field, although, the sound pressure and the acoustic velocity have high values. This means that the reactive intensity is high. In the far-field, the sound pressure and the acoustic velocity are in phase and the vector direction of the IAI only points out from the sound source.

The integral of the IAI over the period of the acoustic signal $T$ leads to the time-averaged acoustic intensity AAI, following Equation (9). In Figure 4, the contour denotes the AAI level. Due to the source motion, the acoustic intensity is distributed along the path of the source. The characteristic of the acoustic intensity propagation is analyzed in the $x y$-plane in Figure 4a and in the $x z$-plane in Figure $4 \mathrm{~b}$. The region inside the source rotation $\left(r<\rho_{\mathrm{s}}\right)$ is named the inner region and the region outside $\left(r>\rho_{\mathrm{s}}\right)$ is named the outer region. The energy streamlines follow the timeaveraged acoustic intensity vectors.

The acoustic energy flow of a stationary monopole point source is in the radial direction from the source. 


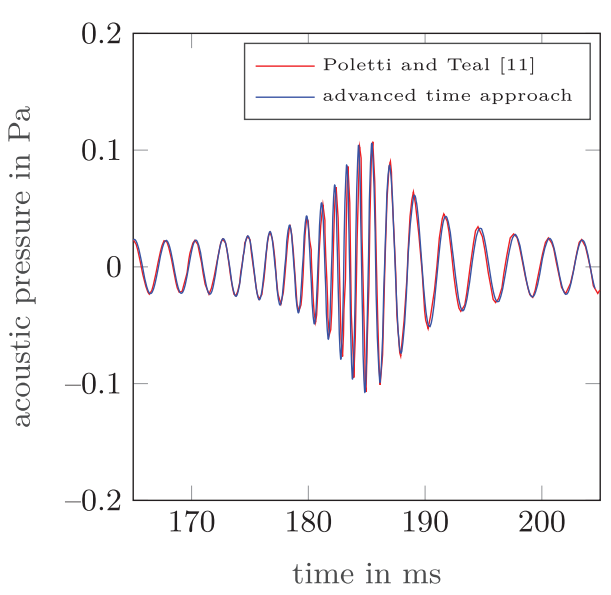

(a)

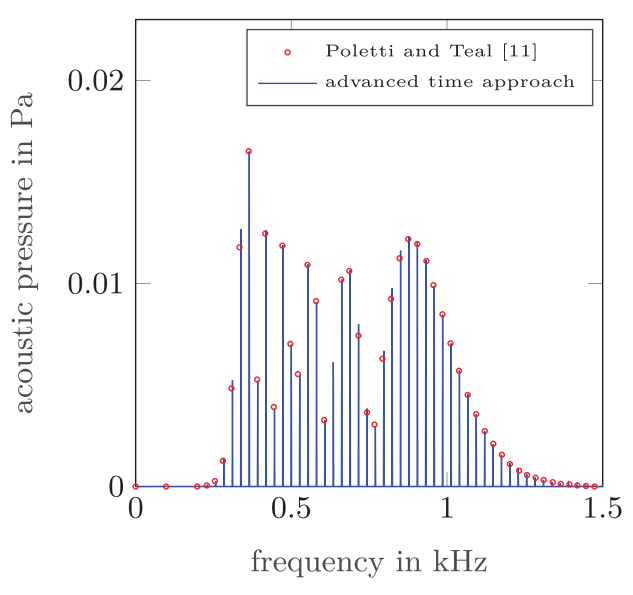

(b)

Figure 1. Acoustic pressure at the observer position $(x, y, z)=(2 \mathrm{~m}, 0 \mathrm{~m}, 0 \mathrm{~m})$ for a sound source rotating in counter-clockwise direction around the $z$-axis on the $x y$-plane at $M_{0} \approx 0.5$. (a) Time signal; (b) Pressure spectrum.

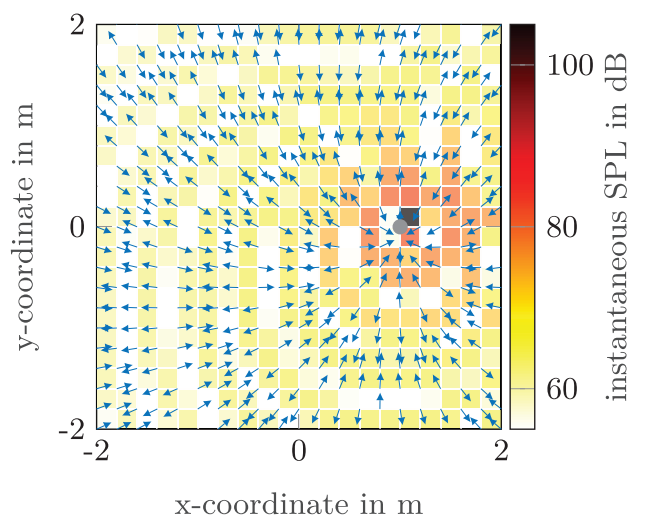

Figure 2. Instantaneous sound pressure level and acoustic velocity vector direction due to a sound source rotating in counter-clockwise direction at $M_{0} \approx 0.5$ at the time $t=0 \mathrm{~s}$. The gray dot marks the instantaneous source position.

For rotating point sources, the flow direction of the timeaveraged acoustic energy differs from the radial direction and depends on the rotational direction and the rotational velocity. In the outer region, the energy flow angle differs from the radial direction by $\delta \approx 10.7^{\circ}$ at a radius of $1.6 \mathrm{~m}$. Changing the rotational direction of the sound source for the considered case, changes the sign of $\delta$. A more detailed analysis of the energy flow angle in the far-field at different rotational velocities is shown in Section 3.3. In the inner region, a part of the acoustic energy flows towards the rotation center and then propagates along the axial direction.

Figure 5 visualizes one-eighth of the three-dimensional AAI field and the energy streamlines. It should be kept in mind, that the acoustic energy does not rotate around the axis of rotation as might be assumed from the projection view in the $x y$-plane. Instead, most of the acoustic energy propagates parallel to the $z$-axis. In contrast to Mao et al. [12], the energy streamlines in Figures 4 and 5 consider all frequency components of the Doppler-shifted acoustic intensity spectrum $\tilde{\mathbf{I}}(\mathbf{x}, \omega)$. Picking one frequency component of the AAI spectrum $\tilde{\mathbf{I}}(\mathbf{x}, \omega)$, we can analyze the energy flow for a specific propagation mode as done by Mao et al. [12]. Figure 6 shows the AAI field for the frequency components, which correspond to the propagation modes $m=-5,0,+5$. The discrete frequencies which correspond to the propagation modes are calculated with $f_{m}=$ $\mathrm{f}_{0}+m f_{\mathrm{R}}$. These propagation modes correspond to the spinning modes in the SHSEM. The contour denotes the AAI level for the frequency component and the energy streamlines follow the acoustic intensity vectors. For the propagation mode $m=0$, the direction of the acoustic energy flow is radial in the inner and the outer region. The flow of the acoustic energy for the negative propagation modes is in the opposite direction as the rotation direction of the sound source (Fig. 6b), whereas, it flows in the same direction for positive propagation modes (Fig. 6c). Further, the AAI level for negative propagation modes is more dominant in the outer region compared to the AAI level for positive propagation modes, which is more dominant in the inner region.

\subsection{Supersonic rotation}

For the supersonic case, the rotation frequency is increased to $f_{\mathrm{R}}=81.2 \mathrm{~Hz}$ and the frequency of the sound source remains at $f_{0}=500 \mathrm{~Hz}$, which corresponds to a rotational Mach number of $M_{0}=2 \pi f_{\mathrm{R}} \rho_{\mathrm{s}} / c_{0} \approx 1.5$. For sound sources rotating at high velocities (typically, but not necessarily, supersonic velocities) negative rotationally Dopplershifted frequencies occur for $f_{0}<m f_{\mathrm{R}}$. For the supersonic case, Poletti and Teal did not provide results from the advanced time approach, hence, we compare the pressure signal and the pressure spectrum with those calculated with the SHSEM. For the reconstruction of the waveform, the source data of 19 periods of rotation are analyzed with a sampling frequency of $f_{\mathrm{s}}=1 \times 10^{6} \mathrm{~Hz}$. The frequency resolution is $6.76 \mathrm{~Hz}$. This frequency resolution has been chosen 


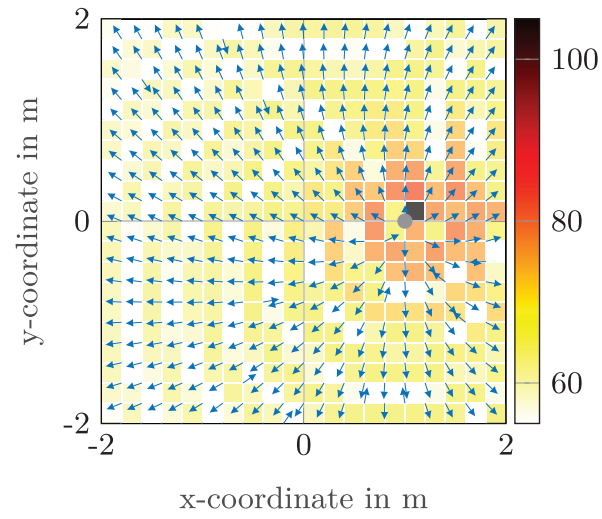

(a)

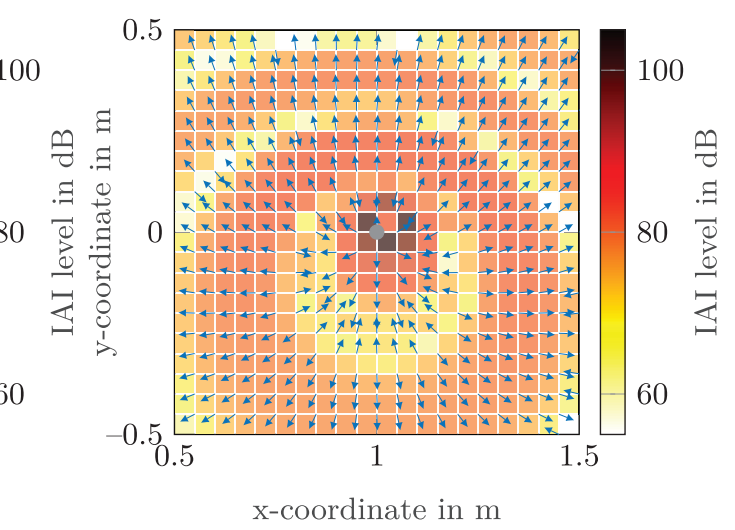

(b)

Figure 3. Instantaneous acoustic intensity due to a sound source rotating in counter-clockwise direction at $M_{0} \approx 0.5$ at the time $t=0 \mathrm{~s}$. The gray dot marks the instantaneous source position. (a) IAI level and IAI vector field; (b) Close-up of the near-field IAI.

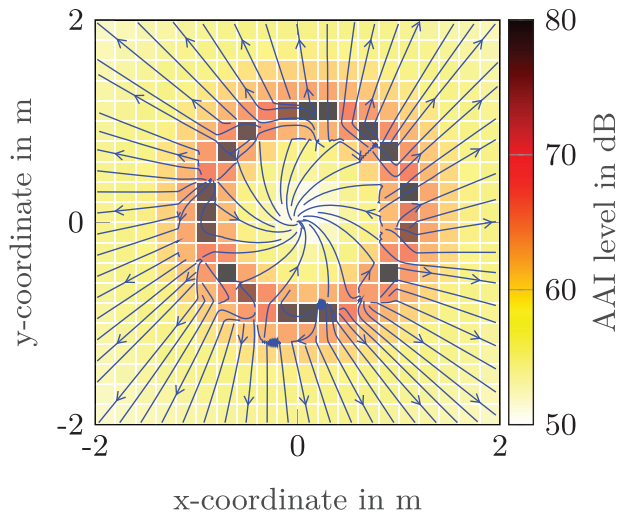

(a)

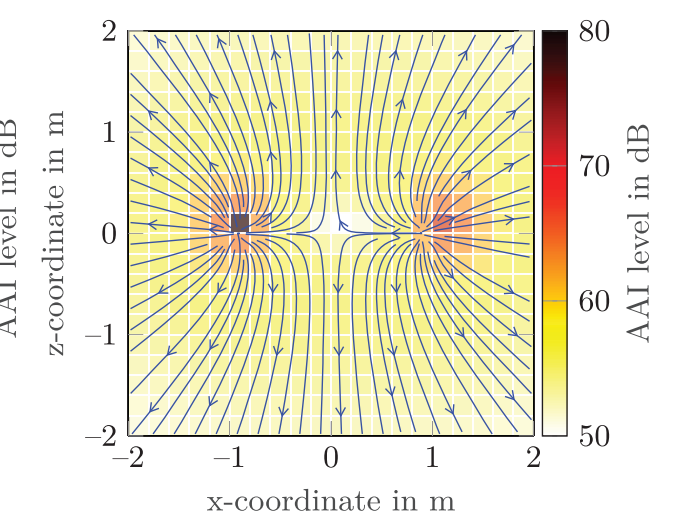

(b)

Figure 4. Time-averaged acoustic intensity level and streamlines of the acoustic energy flow due to a sound source rotating at $M_{0} \approx 0.5$ in counter-clockwise direction. (a) $x y$-plane; (b) $x z$-plane.

to resolve the propagation modes correctly. Further, a lowpass Butterworth filter of fourth-order was applied on the time signals [21]. The cut-off frequency was chosen to $f_{\text {cut }}=4 \times 10^{4} \mathrm{~Hz}$, which corresponds to the 500 resolved propagation modes used for the calculation with the SHSEM in Poletti and Teal [11]. Figure 7a shows a close-up of one occurring shock wave. The advanced time approach produces the same waveform as the SHSEM.

The waveform is reconstructed well for the supersonic case with the advanced time approach. Following Poletti and Teal [11], the maximum spectral frequency for sources at subsonic velocity is estimated as $f_{0} /\left(1-M_{0}\right)$. Considering $M_{0}=1$, the maximum spectral frequency is infinite. This is also valid for sound sources at supersonic rotation, since there are source positions relative to the observer position with $M_{0}=1$. However, we obtain a reliable pressure spectrum and energy flow angle by applying a low-pass filter on the time signal and increasing the sampling rate. Due to the high sampling rate, aliasing effects can be minimized for the analyzed frequency range from 0 to $40 \mathrm{kHz}$.

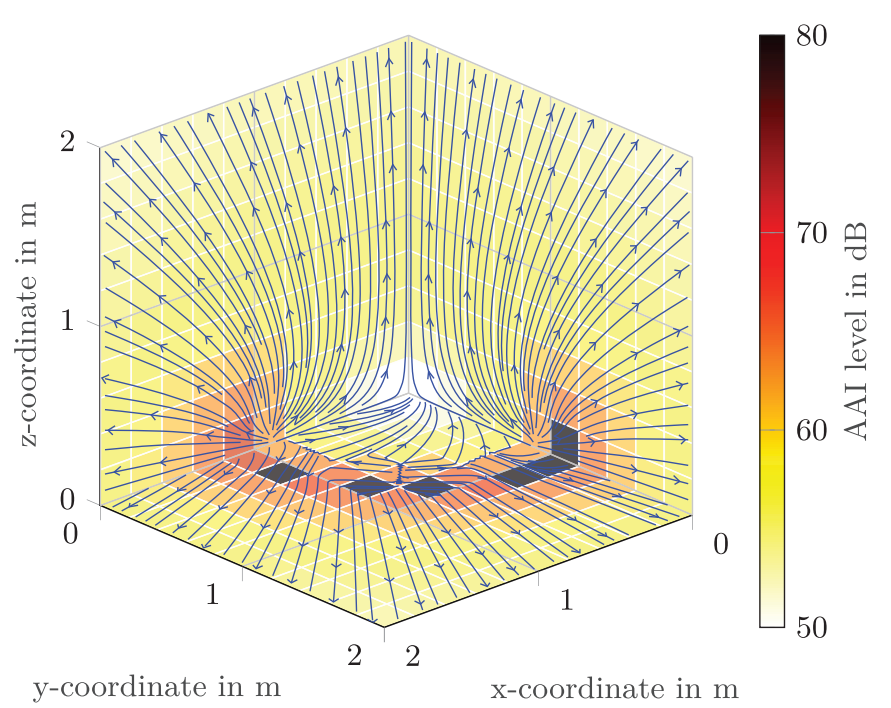

Figure 5. Three-dimensional AAI field and streamlines of the acoustic energy flow around a point source rotating at $M_{0} \approx 0.5$. 


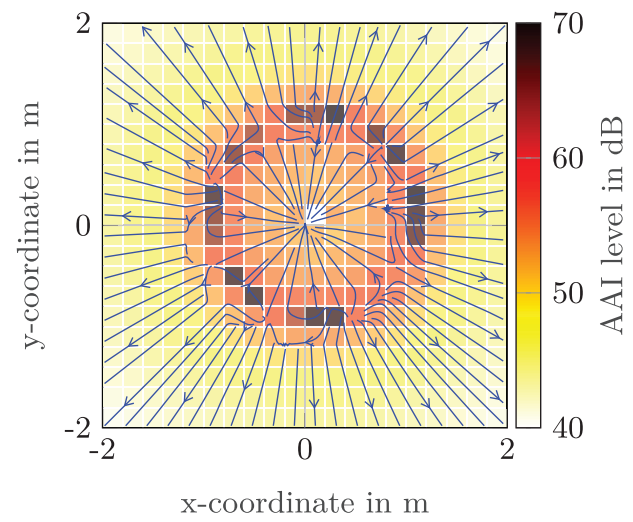

(a)

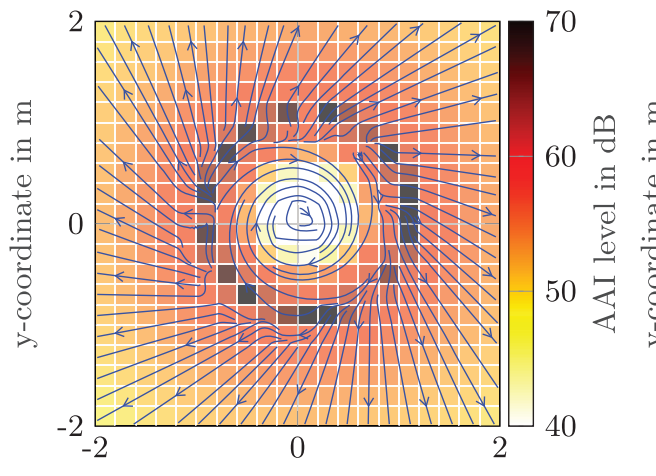

$\mathrm{x}$-coordinate in $\mathrm{m}$

(b)

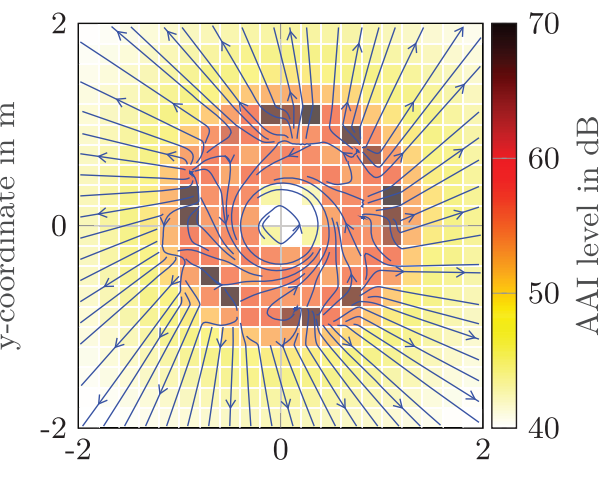

$\mathrm{x}$-coordinate in $\mathrm{m}$

(c)

Figure 6. Frequency components of the AAI spectrum. AAI level and streamlines of the acoustic energy flow due to a sound source rotating at $M_{0} \approx 0.5$ in counter-clockwise direction. (a) $m=0: f_{\mathrm{m}}=500 \mathrm{~Hz}$; (b) $m=-5: f_{\mathrm{m}}=365 \mathrm{~Hz} ;(\mathrm{c}) m=+5: f_{\mathrm{m}}=635 \mathrm{~Hz}$.

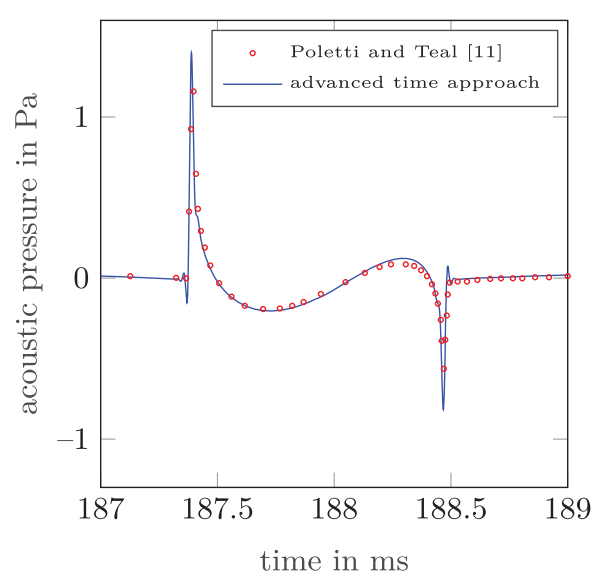

(a)

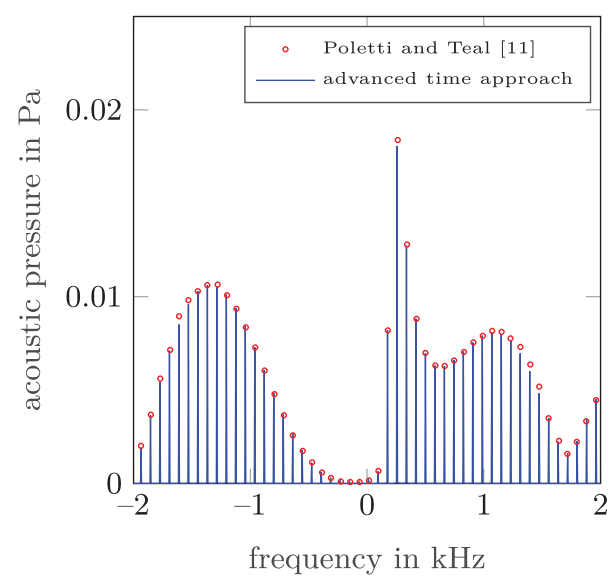

(b)

Figure 7. Acoustic pressure at the observer position $(x, y, z)=(2 \mathrm{~m}, 0 \mathrm{~m}, 0 \mathrm{~m})$ for a sound source rotating in counter-clockwise direction around the $z$-axis on the $x y$-plane at $M_{0} \approx 1.5$. (a) Time signal; (b) Pressure spectrum.

It should be kept in mind that the amplitude of the shock wave is truncated by the cut-off frequency for the advanced time approach and by the number of resolved propagation modes for the SHSEM. A higher cut-off frequency increases only the amplitude of the shock front, whereas the rest of the time signal remains unchanged. These high sampling 


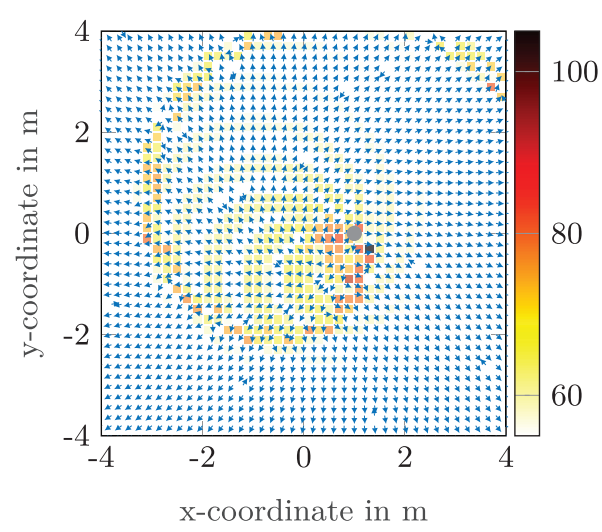

(a)

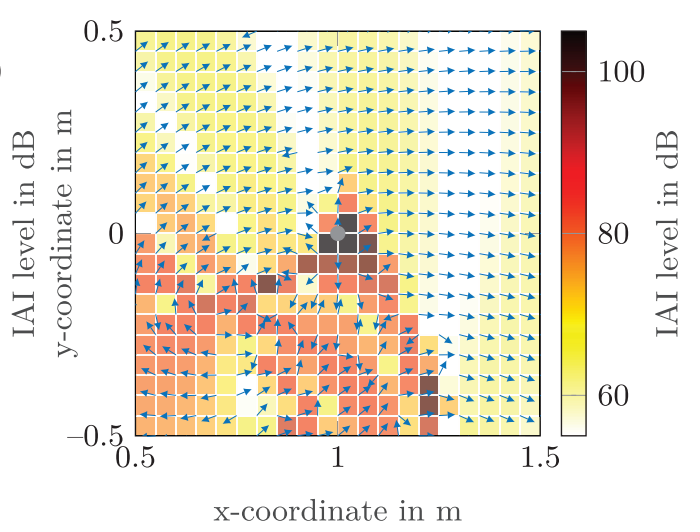

(b)

Figure 8. Instantaneous acoustic intensity due to a sound source rotating in counter-clockwise direction at $M_{0} \approx 1.5$ at the time $t=0 \mathrm{~s}$. The gray dot marks the instantaneous source position. (a) IAI level and IAI vector field; (b) Close-up of the near-field IAI.

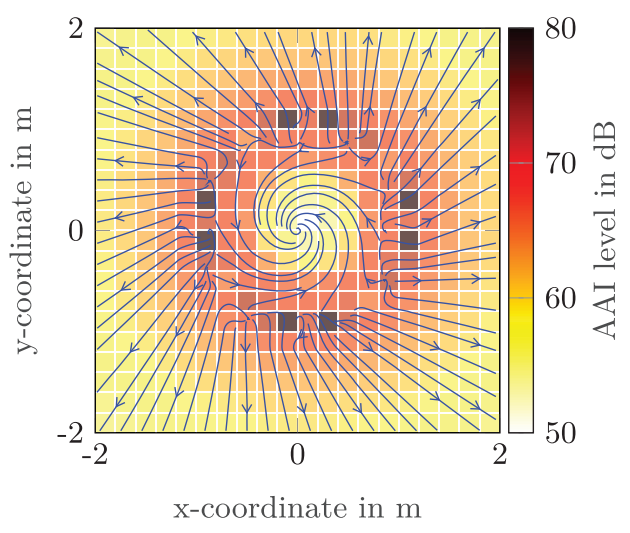

(a)

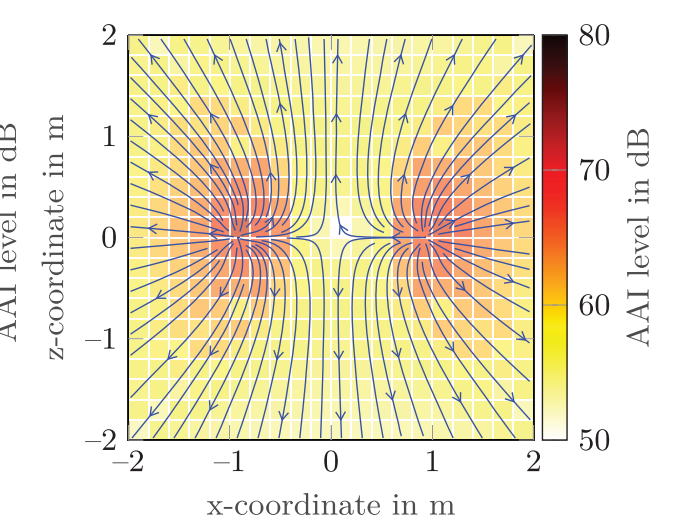

(b)

Figure 9. Time-averaged acoustic intensity level and streamlines of the acoustic energy flow due to a sound source rotating at $M_{0} \approx 1.5$ in counter-clockwise direction. (a) $x y$-plane; (b) $x z$-plane.

rates and the low-pass filter are also needed for the retarded time approach to resolve the shock front.

The contour of the IAI level and the IAI vector field are given in Figure 8. In Figure 8a, the observed area is increased to $8 \mathrm{~m} \times 8 \mathrm{~m}$ for a better visualization of the curved shock wave front.

For the supersonic case, a curved shock wave front is formed that is seen to spiral outward from the source position. As it is well known in the case of supersonically linear motion of a sound source, a Mach cone forms. When the source travels on a circular path, the Mach cone is radically different. Near the source, the cone is similar to the linear case (see Fig. 8b), but to greater radii, a cusp is formed. The cusp singularity is produced at the position $r_{\text {cusp }}=$ $\rho_{\mathrm{s}} / M_{0}=0.67 \mathrm{~m}$. A more detailed discussion on the Mach cone at circular motion is presented by Lowson and Jupe [22], Poletti [7], and Ocker and Pannert [23].

Figure 9 visualizes the time-averaged active acoustic intensity field and the acoustic energy propagation, which follows the active intensity, for the supersonic case.
The sound field looks similar to the subsonic case. However, the angle of the energy flow is increased to $\delta \approx 20.6^{\circ}$ in the outer region. In the inner region, the energy streamlines in the $x y$-plane are more twisted towards the axis of rotation compared to the subsonic case in Figure 4a.

\subsection{Direction of the acoustic energy flow}

The direction of the acoustic energy flow depends on the direction of rotation, the rotational velocity of the sound source and the observer position. For the analysis, we define the acoustic energy flow angle as the inclined angle between the AAI vector direction and the positive radial propagation direction. A sampling frequency of $f_{\mathrm{s}}=1 \times 10^{6} \mathrm{~Hz}$ is used and a low-pass Butterworth filter with a cut-off frequency of $f_{\mathrm{s}}=4 \times 10^{4} \mathrm{~Hz}$ is applied to obtain a convergence of the acoustic flow angle up to $M_{0}=2$. The further numerical setup is the same as for the shown cases above.

First, we analyze the flow angle as a function of the radius, exemplarily shown for $M_{0}=0.5, M_{0}=1.0$ and 


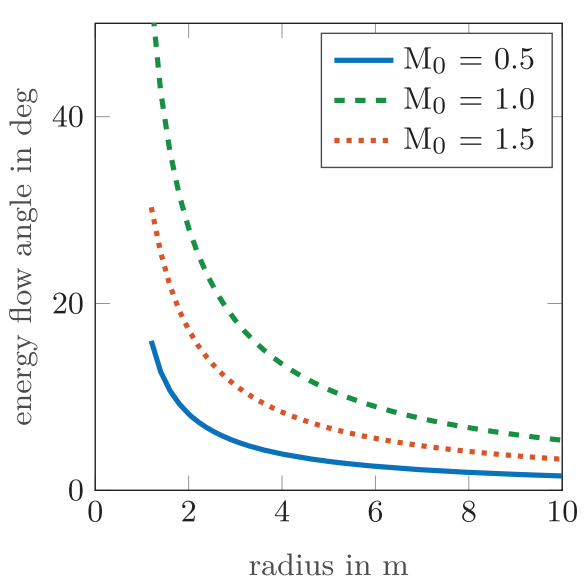

(a)

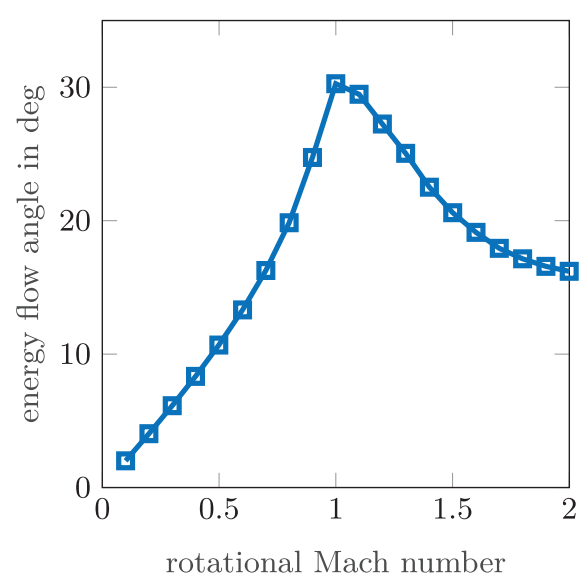

(b)

Figure 10. Acoustic energy flow angle relative to the radial direction. (a) Acoustic energy flow angle as a function of the radius; (b) Acoustic energy flow angle as a function of the rotational velocity on a radius of $1.6 \mathrm{~m}$.

$M_{0}=1.5$ (Fig. 10a). In the near-field outer region, the flow angle decreases fast with increasing radius, whereas in the far-field outer region, it decreases slowly towards the radial propagation direction. The energy flow angle for a rotational velocity of $M_{0}=1.0$ is higher for all radii compared to $M_{0}=1.5$. As a second step, we evaluate the flow angle on the fixed radius of $1.6 \mathrm{~m}$ as a function of the rotational velocity. As can be seen in Figure 10b, the angle of the acoustic energy flow relative to a radial direction increases for higher rotational velocities up to $M_{0}=1.0$. Above $M_{0}=1.0$, the angle reduces for higher Mach numbers.

The angle of the acoustic intensity vector is important to improve the absorption performance of acoustic liners and duct absorbers of axial fans. Therefore, it is reasonable to calculate the angle of the acoustic intensity vector first for a monochromatic rotating sound source. Although the advanced time approach needs high sampling rates, it is computationally less expensive than calculating several hundred propagation modes with the SHSEM as Poletti and Teal [11] did in their investigation.

\section{Conclusions}

In comparison to the SHSEM and the retarded time approach, the advanced time approach is an easy to implement and straightforward method for the calculation of the sound fields around rotating sound sources under free-field conditions. Especially for the supersonic case, the calculation is computationally expensive due to the high mode orders with the SHSEM and solving all roots of a nonlinear equation for the retarded time approach. The advanced time approach calculates the three-dimensional sound pressure, acoustic velocity and acoustic intensity fields around sound sources at subsonic and supersonic rotation. The calculated sound fields are reliable for subsonic velocities. For supersonic rotation, the waveform of the pressure signal is reproduced well compared to the other methods, but high sampling rates are needed and a low-pass filter has to be applied to the time signals. A cut-off frequency was chosen, which corresponds to the number of resolved propagation modes with SHSEM. Then, the direction of the acoustic energy flow is derived from the acoustic intensity vectors. For rotating sound sources, the angle of the acoustic energy flow differs from the radial direction and depends on the rotational velocity. The angle of the acoustic energy flow increases for rotational Mach numbers up to $M_{0}=1$ and decreases again for Mach numbers above 1. With these results, sound absorbers can be designed according to the prevailing rotational velocity to increase the acoustic absorption properties in the rotor plane as well as other planes in axial direction.

\section{References}

1. F. Czwielong, S. Floss, M. Kaltenbacher, S. Becker: Influence of a micro-perforated duct absorber on sound emission and performance of axial fans. Applied Acoustics 174 (2021) 107746

2. B. Dong, D. Xie, F. He, L. Huang: Noise attenuation and performance study of a small-sized contra-rotating fan with microperforated casing treatments. Mechanical Systems and Signal Processing 147 (2021) 107086.

3. S. Floss, F. Czwielong, M. Kaltenbacher, S. Becker: Design of an in-duct micro-perforated panel absorber for axial fan noise attenuation. Acta Acustica 5 (2021) 24.

4. D.L. Sutliff, M.G. Jones, T.C. Hartley: High-speed turbofan noise reduction using foam-metal liner over-the-rotor. Journal of Aircraft 50 (2013) 1491-1503.

5.X.Q. Ma, Z.T. Su: Development of acoustic liner in aero engine: a review. Science China Technological Sciences 63 (2020) 2491-2504.

6. M. Carley: Series expansion for the sound field of rotating sources. The Journal of the Acoustical Society of America 120 (2006) 1252-1256.

7. M.A. Poletti: Series expansions of rotating two and three dimensional sound fields. The Journal of the Acoustical Society of America 128 (2010) 3363-3374. 
8. D. Casalino: An advanced time approach for acoustic analogy predictions. Journal of Sound and Vibration 261 (2003) 583-612.

9. K.S. Brentner: An efficent and robust method for predicting helicopter high-speed impulsive noise. Journal of Sound and Vibration 203 (1997) 87-100.

10. F. Farassat: Derivation of formulations 1 and $1 \mathrm{~A}$ of Farassat (NASA/TM-2007-214853) (2007). https://ntrs.nasa.gov/ citations $/ 20070010579$.

11. M. Poletti, P.D. Teal: Comparison of methods for calculating the sound field due to a rotating monopole. The Journal of the Acoustical Society of America 129 (2011) 3513-3520.

12. Y. Mao, C. Xu, D. Qi: Computation of instantaneous and time-averaged active acoustic intensity field around rotating source. Journal of Sound and Vibration 337 (2015) 95-115.

13.J. Prieur: Calculation of transonic rotor noise using a frequency domain formulation. AIAA Journal 26 (1988) $156-162$.

14. D.B. Hanson: Direct frequency domain calculation of open rotor noise. AIAA Journal 30 (1992) 2334-2337.

15. M. Gennaretti, C. Testa, G. Bernardini: Frequency-domain method for discrete frequency noise prediction of rotors in arbitrary steady motion. Journal of Sound and Vibration 331 (2012) 5502-5517.
16. H. Tang, D. Qi, Y. Mao: Analysis on the frequency-domain numerical method to compute the noise radiated from rotating sources. Journal of Sound and Vibration 332 (2013) 6093-6103.

17. S. Haykin, M. Moher: An Introduction to Analog and Digital Communications. Wiley (2006).

18. S. Maymon, A.V. Oppenheim: Sinc interpolation of nonuniform samples. IEEE Transactions on Signal Processing 59 (2011) 4745-4758.

19. F. Jacobsen: A note on instantaneous and time-averaged active and reactive sound intensity. Journal of Sound and Vibration 147 (1991) 489-496.

20. J. Kaiser, R. Schafer: On the use of the I0-sinh window for spectrum analysis. IEEE Transactions on Acoustics, Speech, and Signal Processing 28 (1980) 105-107.

21. S. Butterworth: On the theory of filter amplifiers. Experimental Wireless and the Wireless Engineer 7 (1930) 536-541.

22. M.V. Lowson, R.J. Jupe: Wave forms for a supersonic rotor. Journal of Sound and Vibration 37 (1974) 475-489.

23. C. Ocker, W. Pannert: Acoustic ray method derived with the concept of analogue gravity for the calculation of the sound field due to rotating sound sources. Applied Acoustics 168, 107422 (2020).

Cite this article as: Ocker C. \& Pannert W. 2021. Three-dimensional acoustic energy flow from rotating sound sources. Acta Acustica, 5, 48. 